\title{
UNE SEXTIQUE DE L'ESPACE PROJECTIF RÉEL AVEC UN GRAND NOMBRE D'ANSES
}

\author{
F. BIHAN*
}

\begin{abstract}
Résumé
Il découle des restrictions connues sur la topologie d'une variété algébrique réelle que le nombre d'anses de l'ensemble des points réels d'une sextique réelle non singulière dans $\mathbb{C} P^{3}$ n'excède pas 47 . Nous construisons une sextique réelle non singulière $X_{6}$ dans $\mathbb{C} P^{3}$ dont l'ensemble des points réels $\mathbb{R} X_{6}$ a 44 anses. En particulier, cette surface vérifie $b_{1}\left(\mathbb{R} X_{6}\right)=h^{1,1}\left(X_{6}\right)+2$. Nous étendons la construction afin d'obtenir pour tout entier pair $m \geq 6$ une surface réelle non singulière $X_{m}$ de degré $m$ dans $\mathbb{C} P^{3}$ vérifiant $b_{1}\left(\mathbb{R} X_{m}\right)>$ $h^{1,1}\left(X_{m}\right)$. Il est connu qu'une telle surface n'existe pas si $m \leq 4$.
\end{abstract}

\begin{abstract}
It follows from the known restrictions on the topology of a real algebraic variety that the number of handles of the real part of a real nonsingular sextic in $\mathbb{C} P^{3}$ is at most 47 . We construct a real nonsingular sextic $X_{6}$ in $\mathbb{C} P^{3}$ whose real part $\mathbb{R} X_{6}$ has 44 handles. In particular, this surface verifies $b_{1}\left(\mathbb{R} X_{6}\right)=h^{1,1}\left(X_{6}\right)+2$. We extend the construction in order to obtain for any even $m \geq$ 6 a real nonsingular surface $X_{m}$ of degree $m$ in $\mathbb{C} P^{3}$ verifying $b_{1}\left(\mathbb{R} X_{m}\right)>h^{1,1}\left(X_{m}\right)$. It is known that such a surface does not exist if $m \leq 4$.
\end{abstract}

${ }^{*}$ A part of the present work was done during the doctoral position of the author at the university of Rennes (France), the other part was done during its post-doctoral position at the university of Genève (Suisse). The author thanks both these institutions for their hospitality.

1991 Mathematics Subject Classification: 14P25, 14J99.

Servicio de Publicaciones. Universidad Complutense. Madrid, 2001 


\section{Introduction}

Une surface algébrique $X$ dans $\mathbb{C} P^{3}$ est dite réelle si elle est définie par un polynôme à coefficients réels. Son ensemble des points réels (ou partie réelle) noté $\mathbb{R} X$ est alors la surface algébrique dans $\mathbb{R} P^{3}$ définie par le polynôme. Plus généralement, une variété algébrique réelle est une variété algébrique complexe $X$ equipée d'une involution antiholomorphe $c: X \rightarrow X$. Son ensemble de points réels $\mathbb{R} X$ est l'ensemble des points fixes de $c$. Par la suite les surfaces considérées seront supposées non singulières et on notera $X_{m}$ une surface algébrique réelle de degré $m$ dans $\mathbb{C} P^{3}$. Dans ce papier, nous nous intéréssons au problème de la classification des types topologiques (c.a.d. à homéomorphisme près) des surfaces $\mathbb{R} X_{m}$ de degré $m$ donné. Le premier cas difficile est le cas $m=4$, la question correspondante fut posée par Hilbert au début du XX-ième siècle dans le seizième problème de sa fameuse liste. La réponse finale à cette dernière question fut apportée par $\mathrm{V}$. Kharlamov [K1] en 1976. À ce jour aucun des cas $m \geq 5$ n'est résolu. Il est bien connu qu'une surface $\mathbb{R} X_{m}$ est homéomorphe à une union disjointe de sphères avec ou sans anses (sommes connexes de tores), si $m$ est pair, et d'un plan projectif avec ou sans anses si $m$ est impair. On peut alors étudier le problème, à priori moins difficile que le précédent, des nombres maximaux de composantes connexes et d'anses d'une surface $\mathbb{R} X_{m}$ de degré $m$ donné, ou de manière équivalente, des valeurs maximales des nombres de Betti $b_{0}\left(\mathbb{R} X_{m}\right)$ et $b_{1}\left(\mathbb{R} X_{m}\right)$ pour $m$ donné (où ici et par la suite les groupes d'homologie sont pris à coefficients dans $\mathbb{Z} / 2$ ). Même ces valeurs ne sont pas connues pour $m \geq 5$ (voir, par exemple, [I-K, B1] pour le cas $m=5$, [V2, I, B2, D-K] pour le cas général).

Notre premier résultat concerne le cas $m=6$. Les restrictions classiques imposent que le nombre maximal d'anses d'une surface $\mathbb{R} X_{6}$ ne doit pas dépasser 47 (voir section 1), par ailleurs, la meilleure borne inférieure connue était donnée par une surface $\mathbb{R} X_{6}$ avec 43 anses (voir [V1]). Nous améliorons cette borne inférieure (Théorème 5.1, section 5 ) en construisant une sextique $X_{6}$ dont l'ensemble des points réels est homéomorphe à l'union disjointe $6 S \amalg S_{2} \amalg S_{42}$ de six sphères, d'une sphère avec deux anses et d'une sphère avec quarante deux anses. Le deuxième résultat concerne l'inégalité $b_{1}(\mathbb{R} X) \leq h^{1,1}(X)$ portant sur une surface algébrique réelle $X$ non singulière, simplement connexe et projective (où $h^{p, q}(X)$ est le nombre de Hodge de type $(p, q)$ associé à 
$X)$. Cette inégalité fut proposée par $\mathrm{O}$. Viro comme une généralisation de la conjecture de Ragsdale portant sur les courbes réelles planes. Les premiers contre-exemples à l'inégalité de Viro ont été obtenus par I. Itenberg [I] qui a construit (à partir de ses contre-exemples à la conjecture de Ragsdale) pour tout entier pair $m \geq 10$ une surface $X_{m}$ vérifiant $b_{1}\left(\mathbb{R} X_{m}\right)>h^{1,1}\left(X_{m}\right)$. Nous montrons (Théorème 5.2, section 5)) l'existence de contre-exemples $X_{m}$ pour tout entier pair $m \geq 6$ (le contre-exemple en degré 6 est réalisé par la sextique plus haut). Il découle par ailleurs de la classification connue que l'inégalité de Viro est vraie pour les surfaces $X_{m}$ de degré $m \leq 4$ (voir, par exemple, [D-K]).

Comme dans [I], les surfaces présentées ici sont construites au moyen $\mathrm{du}$ patchwork combinatoire qui est une version combinatoire de la très puissante méthode de Viro de construction de variétés algébriques réelles avec topologie (de la partie réelle) prescrite (voir [V3, V4, R, G-K-Z]). Les variétés réelles ainsi construites sont plongées dans des variétés toriques projectives. Nous décrivons maintenant succintement le patchwork combinatoire. À un polytope convexe $P$ dans $\mathbb{R}^{n}$ (on peut supposer que $P$ est de dimension $n$ ) dont les sommets ont des coordonnées entières correspond une variété torique projective notée $X(P)$. Tout polynôme à coefficients réels et de polytope de Newton $P$ définit une hypersurface algébrique réelle dans $X(P)$. Les données de la construction sont une triangulation "convexe" de $P$ dont les sommets ont des coordonnées entières et une distribution de signes \pm 1 à ces sommets. À partir de ces données et en suivant certaines règles combinatoires, on recolle des morceaux d'hyperplans de $\mathbb{R}^{n}$ afin d'obtenir une variété linéaire par morceaux. On associe par ailleurs aux données un polynôme appelé $T$-polynôme dont le polytope de Newton est $P$, dont les monômes correspondent aux sommets de la triangulation et les signes des monômes aux signes distribués sur les sommets correspondants. Le théorème de Viro affirme en particulier qu'un tel T-polynôme définit une hypersurface algébrique réelle dans $X(P)$, appelée T-hypersurface, dont l'ensemble des points réels est homéomorphe à la variété linéaire par morceaux précédemment construite.

Dans ce papier, on utilise un polytope $Q_{k}$ dans $\mathbb{R}^{3}$ dépendant d'un entier positif $k$ tel que la variété $X\left(Q_{k}\right)$ (qui elle ne dépend pas de $k$ ) soit plongée comme une quadrique dans un espace projectif tordu de dimension quatre. Les surfaces réelles dans $X\left(Q_{k}\right)$ considérées peuvent être 
déformées de manière équivariante, par rapport aux structures réelles, en des surfaces $X_{2 k}$ dans $\mathbb{C} P^{3}$. Les triangulations (et distributions de signes associées) du tétraèdre dans $\mathbb{R}^{3}$ correspondant à la variété torique $\mathbb{C} P^{3}$ qui sont utilisées dans $[\mathrm{I}]$ peuvent être adaptées au polytope $Q_{k}$ afin de produire après déformation des contre-exemples $X_{2 k}$ de plus petits degrés à l'inégalité de Viro.

Ce papier est organisé de la manière suivante. Dans la première section nous présentons les principales restrictions connues sur la topologie de l'ensemble des points réels d'une surface réelle et établissons l'inégalité $b_{1}\left(\mathbb{R} X_{6}\right) \leq 94$. Dans la section 2 nous définissons les surfaces réelles $\mathcal{C}_{k}$ dans la variété torique $X\left(Q_{k}\right)$ qui seront utilisées et montrons l'existence d'une déformation équivariante d'une surface $\mathcal{C}_{k}$ sur une surface $X_{2 k}$. Nous décrivons dans la section 3 le patchwork combinatoire d'une surface $\mathcal{C}_{k}$ et donnons dans la section 4 quelques résultats techniques sur la caractéristique d'Euler de la partie réelle d'une telle surface. Finalement dans la section 5 nous énonçons et prouvons les théorèmes 5.1 et 5.2.

Remerciements. Je voudrais remercier I. Itenberg pour m'avoir proposé le sujet traité dans ce papier.

\section{Restrictions sur la topologie des surfaces algébriques réelles}

Soit $X$ une surface algébrique réelle (non singulière). Nous présentons maintenant les principales restrictions connues sur la topologie de l'ensemble des points réels de $X$. Pour plus de détails, le lecteur pourra par exemple consulter $[\mathrm{K} 2, \mathrm{D}-\mathrm{K}, \mathrm{W}]$. En particulier ces restrictions permettent d'obtenir l'inégalité $b_{1}\left(\mathbb{R} X_{6}\right) \leq 94$ portant sur les sextiques réelles dans $\mathbb{C} P^{3}$.

\section{Inégalité et congruence de Smith-Thom:}

$$
b_{*}(\mathbb{R} X) \leq b_{*}(X), \quad b_{*}(\mathbb{R} X) \equiv b_{*}(X) \bmod 2,
$$

où $b_{*}($. ) est la somme totale des nombres de Betti. La surface $X$ est appelée (M-a)-surface ou $M$-surface selon que $a \neq 0$ ou $a=0$, respectivement, dans l'égalité $b_{*}(\mathbb{R} X)=b_{*}(X)-2 a$.

\section{Inégalités de Comessati:}

$$
2-h^{1,1}(X) \leq \chi(\mathbb{R} X) \leq h^{1,1}(X),
$$


si $X$ est projective et où $\chi(\mathbb{R} X)$ est la caractéristique d'Euler de $\mathbb{R} X$.

Congruence de Rokhlin:

$$
\chi(\mathbb{R} X) \equiv \sigma(X) \bmod 16,
$$

si $X$ est une $M$-surface et où $\sigma(X)$ est la signature de $X$.

Les nombres de Hodge des surfaces algébriques dans $\mathbb{C} P^{3}$ sont bien connus (noter que ces surfaces sont simplement connexes).

$$
h^{1,1}\left(X_{m}\right)=\frac{2 m^{3}}{3}-2 m^{2}+\frac{7 m}{3}, \quad h^{2,0}\left(X_{m}\right)=\frac{m^{3}}{6}-m^{2}+\frac{11 m}{6}-1 .
$$

On a de plus

$$
b_{*}\left(X_{m}\right)=2 h^{2,0}\left(X_{m}\right)+2+h^{1,1}\left(X_{m}\right)=m^{3}+4 m^{2}-6 m,
$$

et

$$
\sigma\left(X_{m}\right)=2 h^{2,0}\left(X_{m}\right)+2-h^{1,1}\left(X_{m}\right)=-\frac{m^{3}}{3}+\frac{4 m}{3} .
$$

En sommant l'inégalité de Smith-Thom et l'une des inégalités de Comessati on obtient

$$
b_{1}\left(\mathbb{R} X_{m}\right) \leq h^{1,1}\left(X_{m}\right)+h^{2,0}\left(X_{m}\right)=\frac{5 m^{3}}{6}-3 m^{2}+\frac{25 m}{6}-1,
$$

une inégalité similaire pour $b_{0}\left(\mathbb{R} X_{m}\right)$ s'obtient en utilisant l'autre inégalité de Comessati.

Considérons maintenant le cas des sextiques réelles dans $\mathbb{C} P^{3}$. L'inégalité précédente se traduit par $b_{1}\left(\mathbb{R} X_{6}\right) \leq 96$. Une sextique $X_{6}$ vérifiant $b_{1}\left(\mathbb{R} X_{6}\right)=96$ serait une $\mathrm{M}$-surface pour laquelle $\chi\left(\mathbb{R} X_{6}\right)=$ -84 et $\sigma\left(X_{6}\right)=-64$ : on obtiendrait une contradiction avec la congruence de Rokhlin. La congruence de Smith-Thom implique alors $b_{1}\left(\mathbb{R} X_{6}\right) \leq 94$.

\section{Le polytope $Q_{k}$, la variété torique $X\left(Q_{k}\right)$ et une déformation équivariante de surfaces réelles dans $X\left(Q_{k}\right)$}

À partir de maintenant par polytope (ou polygone, simplexe,...) on entendra polytope convexe à sommets entiers dans $\mathbb{R}^{n}$ (un point de $\mathbb{R}^{n}$ est 
F. BIHAN UNE SEXTique DE L'ESPACE PRoJeCtif RÉEL AVEC UN GRAND ...

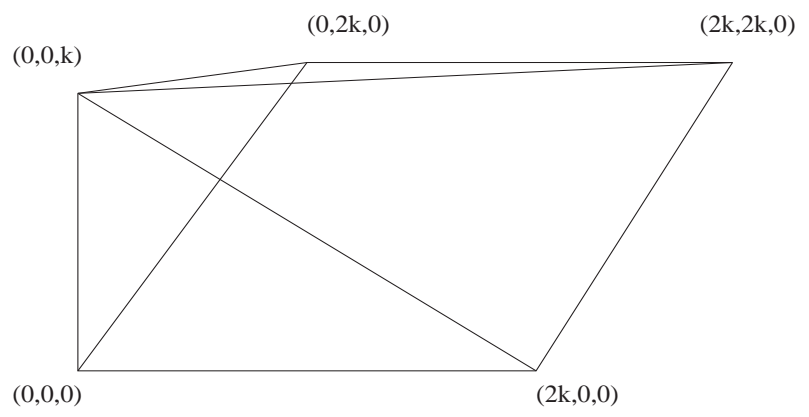

Figure 1: Le polytope $Q_{k}$.

dit entier si ses coordonnées sont entières). Il existe une correspondance $P \rightarrow X(P)$ associant à tout polytope une variété torique projective (voir [G-K-Z]). Cette correspondance n'est pas injective, par exemple tous les homothétiques (de la forme a.P avec a entier) et les translatés (de la forme $b+P$ avec $b$ point entier) de $P$ correspondent à la même variété $X(P)$. Si $\left\{v_{0}, \cdots, v_{s}\right\}$ est un ensemble de points entiers dont l'enveloppe convexe est $P$ alors la variété $X(P)$ se plonge dans $\mathbb{C} P^{s}$ comme la clôture de Zarisky de l'ensemble $\left\{\left(x^{v_{0}}: \cdots: x^{v_{s}}\right), x \in\left(\mathbb{C}^{*}\right)^{n}\right\}$. Soit $f \in \mathbb{C}[x]$ un polynôme de polytope de Newton $P$ (ici on suppose que $P$ est dans l'orthant positif de $\left.\mathbb{R}^{n}\right)$. En prenant pour $\left\{v_{0}, \cdots, v_{s}\right\}$ l'ensemble des exposants des monômes non nuls de $f$, le polynôme $f$ se réduit alors à une forme linéaire sur la variété $X(P)$ plongée comme précédemment dans $\mathbb{C} P^{s}$ et donc définit une hypersurface $Z(f)$ de $X(P)$. La variété $X(P)$ est réelle pour la conjugaison complexe usuelle sur $\mathbb{C} P^{s}$, son ensemble de points réels est une clôture de $\left\{\left(x^{v_{0}}: \cdots: x^{v_{s}}\right), x \in\left(\mathbb{R}^{*}\right)^{n}\right\}$. Si le polynôme $f$ considéré plus haut a des coefficients réels, alors $Z(f)$ est une hypersurface réelle de $X(P)$.

Le polytope $Q_{k}$ que nous utiliserons par la suite est le polytope de sommets $(0,0,0),(2 k, 0,0),(2 k, 2 k, 0),(0,2 k, 0),(0,0, k)$ dans $\mathbb{R}^{3}$ où $k$ est un entier positif donné. La variété $\Theta=X\left(Q_{k}\right)$ correspondante (elle ne dépend pas de $k$ d'après ce qui a été dit plus haut) se plonge dans l'espace projectif tordu $\mathbb{C} P^{4}(2)$ de variables homogènes $Z_{0}, Z_{1}, Z_{2}, Z_{3}$ de poids un et $Z_{4}$ de poids deux comme la quadrique $\left\{Z_{0} Z_{3}-Z_{1} Z_{2}=0\right\}$. Pour le voir, on peut procéder au changement de coordonnées $y_{3}^{\prime}=2 y_{3}, y_{2}^{\prime}=y_{2}, y_{1}^{\prime}=y_{1}$ de $\mathbb{R}^{3}$. Dans les nouvelles 
coordonnées $\left(y_{1}^{\prime}, y_{2}^{\prime}, y_{3}^{\prime}\right)$, le polytope $Q_{k}$ est un polytope $\Delta_{k}$ de sommets $(0,0,0),(2 k, 0,0),(2 k, 2 k, 0),(0,2 k, 0),(0,0,2 k)$. On obtient alors un plongement de $X\left(\Delta_{k}\right)$ dans $\mathbb{C} P^{4}$ comme l'ensemble $\left\{Z_{0}^{\prime} Z_{3}^{\prime}-Z_{1}^{\prime} Z_{2}^{\prime}=0\right\}$ (où $Z_{0}^{\prime}, Z_{1}^{\prime}, Z_{2}^{\prime}, Z_{3}^{\prime}, Z_{4}^{\prime}$ sont des coordonnées homogènes de $\mathbb{C} P^{4}$ ). Il reste à voir que le changement de variables plus haut nous donne un quotient $\mathbb{C} P^{4} \rightarrow \mathbb{C} P^{4}(2), Z_{i}^{\prime} \rightarrow Z_{i}$ envoyant $X\left(\Delta_{k}\right)$ sur $\Theta$.

Plongeons comme précédemment $\Theta$ comme la quadrique $\left\{Z_{0} Z_{3}-\right.$ $\left.Z_{1} Z_{2}=0\right\}$ dans $\mathbb{C} P^{4}(2)$ et considérons une surface réelle dans $\Theta$ définie dans $\mathbb{C} P^{4}(2)$ par un système d'équations du type

$$
\left\{Z_{0} Z_{3}-Z_{1} Z_{2}=0, Z_{4}^{k}+f_{2}(Z) Z_{4}^{k-1}+\cdots+f_{2 i}(Z) Z_{4}^{k-i}+\cdots+f_{2 k}(Z)=0\right\},
$$

où pour simplifier $Z$ désigne le quadruplet de variables homogènes $Z_{0}$, $Z_{1}, Z_{2}, Z_{3}$ et les $f_{2 i}(Z), i=1, \cdots, k$, sont des polynômes à coefficients réels homogènes de degrés $2 i$ en $Z$. Une telle surface sera notée $\mathcal{C}_{k}$. Montrons maintenant qu'une surface $\mathcal{C}_{k}$ générique est définie par un polynôme affine de polytope de Newton $Q_{k}$. Pour cela, considérons la carte affine $\left\{Z_{0} \neq 0\right\}$ de $\mathbb{C} P^{4}(2)$ munie des coordonnées affines $\left(z_{0}, z_{1}, z_{2}, z_{3}, z_{4}\right)$ avec $z_{i}=\frac{Z_{i}}{Z_{0}}$ pour $i=1,2,3$ et $z_{4}=\frac{Z_{4}}{Z_{0}{ }^{2}}$. L'anneau réel de coordonnée de $\Theta$ dans cette carte est $\mathbb{R}\left[x_{1}, x_{2}, x_{3}\right]=$ $\mathbb{R}\left[z_{1}, z_{2}, z_{3}, z_{4}\right] /\left(z_{3}-z_{1} z_{2}\right)$ où $x_{1}, x_{2}, x_{3}$ sont respectivement les images de $z_{1}, z_{2}, z_{4}$ par l'application quotient. Il est facile de voir que les équations de $\mathcal{C}_{k}$ se réduisent dans la carte affine à une équation $g\left(x_{1}, x_{2}, x_{3}\right)=0$ où $g \in \mathbb{R}\left[x_{1}, x_{2}, x_{3}\right]$ a pour polytope de Newton $Q_{k}$. Réciproquement, tout polynôme de polytope de Newton $Q_{k}$ définit une surface $\mathcal{C}_{k}$. Remarquons que la variété $\Theta$ a le point $\left(Z: Z_{4}\right)=(0: 1)$ comme singularité mais que ce dernier point n'appartient à aucune surface $\mathcal{C}_{k}$.

Proposition 2.1. Pour toute surface $\mathcal{C}_{k}$ non singulière dans $\Theta$, il existe une déformation équivariante de $\mathcal{C}_{k}$ sur une surface $X_{2 k}$ dans $\mathbb{C} P^{3}$.

Preuve. On considère la famille de surfaces $\mathcal{C}_{k}^{\epsilon}, \epsilon \in \mathbb{R}, \mathcal{C}_{k}^{0}=\mathcal{C}_{k}$, definies dans $\mathbb{C} P^{4}(2)$ par les équations $\left\{Z_{0} Z_{3}-Z_{1} Z_{2}=\epsilon Z_{4}, Z_{4}^{k}+\cdots+\right.$ $\left.f_{2 i}(Z) Z_{4}^{k-i}+\cdots+f_{2 k}(Z)=0\right\}$. La projection $\mathbb{C} P^{4}(2) \backslash\{(0: 1)\} \rightarrow \mathbb{C} P^{3}$ : $\left(Z: Z_{4}\right) \rightarrow Z$ est bien définie sur $\mathcal{C}_{k}^{\epsilon}$. Si $\epsilon \neq 0$, cette projection réalise un isomorphisme équivariant entre $\mathcal{C}_{k}^{\epsilon}$ et la surface réelle dans $\mathbb{C} P^{3}$ définie par le polynôme $\left(\frac{Z_{0} Z_{3}-Z_{1} Z_{2}}{\epsilon}\right)^{k}+\cdots+f_{2 i}(Z)\left(\frac{Z_{0} Z_{3}-Z_{1} Z_{2}}{\epsilon}\right)^{k-i}+\cdots+f_{2 k}(Z)$. Si la surface $\mathcal{C}_{k}$ est non singulière alors pour $\epsilon \neq 0$ suffisamment petit la surface réelle dans $\mathbb{C} P^{3}$ obtenue par déformation est non singulière. 
La déformation précédente apparait dans $[\mathrm{H}]$ appliquée aux sextiques complexes.

\section{Patchwork combinatoire d'une surface $\mathcal{C}_{k}$}

Soit $\mathcal{R}^{n}$ le groupe des symétries de $\mathbb{R}^{n}$ engendré par les réflections par rapport aux hyperplans de cordonnées. On identifiera selon les cas la symétrie $\left.\left(y_{1}, \cdots, y_{n}\right) \rightarrow\left((-1)^{r_{1}} y_{1}, \cdots,(-1)^{r_{n}} y_{n}\right)\right)$ avec le point entier $r=\left(r_{1}, \cdots, r_{n}\right)$ ou avec $\bar{r} \in(\mathbb{Z} / 2)^{n}$ (si $a$ est un point entier dans $\mathbb{R}^{n}$ on note $\bar{a}$ son image dans $\left.(\mathbb{Z} / 2)^{n}\right)$.

Les données du patchwork combinatoire d'une surface $\mathcal{C}_{k}$ sont une triangulation $\tau$ à sommets entiers du polytope $Q_{k}$ ainsi qu'une distribution de signes $D: V(\tau) \rightarrow \pm 1$ sur l'ensemble $V(\tau)$ des sommets de $\tau$.

Considérons le polytope $Q_{k}^{*}$ de sommets $( \pm 2 k, \pm 2 k, 0),(0,0, \pm k)$ formé des huit copies symétriques de $Q_{k}$ par $\mathcal{R}^{3}$. Notons $F_{1}$ la face de $Q_{k}$ ayant pour sommets $(2 k, 0,0),(2 k, 2 k, 0),(0,0, k)$ et $F_{2}$ celle ayant pour sommets $(0,2 k, 0),(2 k, 2 k, 0),(0,0, k)$. Les faces de dimension deux de $Q_{k}^{*}$ sont les copies symétriques de $F_{1}$ et $F_{2}$ par $\mathcal{R}^{3}$. Étendons $\tau$ en une triangulation $\tau^{*}$ de $Q_{k}^{*}$ invariante par $\mathcal{R}^{3}$. Étendons la distribution de signes $D$ en une distribution de signes sur les sommets de $\tau^{*}$ en suivant la règle $D(r(v))=D(v) \cdot(-1)^{\langle r, v\rangle}$ si $r \in \mathcal{R}^{3}$ et où $\langle$,$\rangle est le produit$ scalaire usuel. Maintenant pour tout tétraèdre de $\tau^{*}$ dont au moins deux sommets ont des signes différents, sélectionnons le triangle ou quadrangle joignant les milieux des arêtes dont les sommets ont des signes différents. L'union de toutes les pièces ainsi sélectionnées est une surface linéaire par morceaux $S_{k}^{*}$ dans $Q_{k}^{*}$. Considérons l'espace $\tilde{Q}_{k}$ obtenu à partir de $Q_{k}^{*}$ en identifiant chacun des points situé sur une copie symétrique de $F_{1}\left(\right.$ resp. $\left.F_{2}\right)$ avec son image par la symétrie $(1,0,0)$ (resp. $\left.(0,1,0)\right)$ de $\mathcal{R}^{3}$. L'espace $\tilde{Q}_{k}$ est homéomorphe à $\mathbb{R} \Theta$ (voir [G-K-Z]). L'image de $S_{k}^{*}$ dans $\tilde{Q}_{k}$ est une surface linéaire par morceaux que l'on note $\tilde{S}_{k}$.

Une subdivision polyédrale d'un polytope $P$ de dimension $n$ est dite convexe si il existe une application convexe $\nu: P \rightarrow \mathbb{R}$ qui soit affine sur chacun des $n$-polytopes de la subdivision mais non affine sur l'union de deux quelconques de ces $n$-polytopes. 
Supposons la triangulation $\tau$ convexe. On forme alors le T-polynôme affine

$$
f_{t}(x)=\sum_{v \in V(\tau)} D(v) t^{\nu(v)} x^{v}
$$

où $t$ est un paramètre réel, $\nu$ est une fonction certifiant la convexité de $\tau$ et $x^{v}=x_{1}^{v_{1}} x_{2}^{v_{2}} x_{3}^{v_{3}}$ si $x=\left(x_{1}, x_{2}, x_{3}\right)$ et $v=\left(v_{1}, v_{2}, v_{3}\right)$. Ce polynôme a pour polytope de Newton $Q_{k}$ et définit donc une surface $\mathcal{C}_{k}$ (qui peut être singulière). Comme cas particulier du théorème de Viro relatif à sa méthode de construction de variétés algébriques réelles, on obtient le résultat suivant.

Proposition 3.1. Pour $t>0$ suffisamment petit, le polynôme $f_{t}$ définit une surface $\mathcal{C}_{k}$ non singulière et il existe un homéomorphisme $\mathbb{R} \Theta \rightarrow \tilde{Q}_{k}$ envoyant $\mathbb{R} \mathcal{C}_{k}$ sur $\tilde{S}_{k}$.

Une surface définie par un T-polynôme est appelée T-surface.

\section{Caractéristique d'Euler de la partie réelle d'une T-surface $\mathcal{C}_{k}$}

On peut calculer la caractéristique d'Euler de la partie réelle d'une Tsurface $\mathcal{C}_{k}$ en appliquant la formule d'Euler à la surface linéaire par morceaux $\tilde{S}_{k}$ correspondante. Les cellules de dimension $p-1$ de $\tilde{S}_{k}$ sont contenues dans les simplexes de dimension $p$ de $\tau^{*}$ dont au moins deux sommets ont des signes différents (un tel simplexe sera dit non vide). On peut alors dans certains cas exprimer de manière relativement simplement la caractéristique d'Euler recherchée en fonction des triangulation et distribution de signes utilisées.

Soit $P$ un polytope dans $\mathbb{R}^{n}$ (rappelons que dans ce papier polytope signifie polytope convexe à sommets entiers). Dans tout ce qui suit la forme volume de $\mathbb{R}^{n}$ considérée sera la forme vol définie par $\operatorname{vol}(\cdot)=$ $n ! \operatorname{Vol}(\cdot)$ où $V o l$ est la forme volume euclidienne usuelle. En particulier le volume $\operatorname{vol}(P)$ de $P$ est un entier positif. On note $|P|$ le plus grand (pour l'inclusion) ensemble de points entiers dont $P$ est l'enveloppe convexe. Un simplexe $\sigma$ est dit maximal si $|\sigma|$ est exactement l'ensemble de ses sommets et primitif si $\operatorname{vol}(\sigma)=1$ (primitif entraine maximal mais la réciproque est fausse en général si $n \geq 3$ ). Une triangulation à sommets entiers d'un polytope $P$ est dite maximale (resp. primitive) si 
tous ses simplexes sont maximaux (resp. primitifs). Une triangulation de $P$ est maximale si et seulement si son ensemble de sommets est $|P|$ tout entier.

Soit $\sigma$ un $p$-simplexe contenu dans l'orthant positif $\left(\mathbb{R}_{+}\right)^{n}$ de $\mathbb{R}^{n}$. On appellera plus simplement copie symétrique de $\sigma$ l'image de $\sigma$ par un élément de $\mathcal{R}^{n}$. Considérons une distribution de signes $D$ aux sommets de $\sigma$ et étendons la en une distribution de signes sur les sommets des copies symétriques de $\sigma$ en suivant la même règle que dans la section 3: $D(r(v))=D(v) \cdot(-1)^{\langle r, v\rangle}$, si $r \in \mathcal{R}^{n}$ et $v$ est un sommet de $\sigma$, ou de manière équivalente,

$$
d(r(v)) \equiv d(v)+\langle r, v\rangle \bmod 2,
$$

si $d(v) \in\{0,1\}$ est défini par $D(v)=(-1)^{d(v)}$. Une copie symétrique de $\sigma$ est dite vide si tous ses sommets ont le même signe, sinon on dit qu'elle est non vide. Soient $v_{0}, \ldots, v_{p}$ les sommets de $\sigma$. Notons $\mathcal{M}_{\sigma}$ la $(p \times n)$-matrice à coefficients dans $\mathbb{Z} / 2$ formée des vecteurs lignes $\overline{v_{i}-v_{0}}$ pour $i=1, \cdots, p$. Il est facile de voir à partir de la règle plus haut qu'une copie symétrique $r(\sigma)$ est vide si et seulement si $\bar{r}$ est solution du système linéaire à coefficients dans $\mathbb{Z} / 2$

$$
\mathcal{M}_{\sigma} \cdot r=d_{\sigma}, \quad(\star),
$$

si $d_{\sigma}=\left(d_{1}, \cdots, d_{p}\right)$ avec $d_{i}=\overline{d\left(v_{i}\right)}+\overline{d\left(v_{0}\right)}$. On dit que $\sigma$ est élémentaire si la matrice $\mathcal{M}_{\sigma}$ est de rang maximal $p$. En particulier si $\sigma$ est de dimension maximale (c.a.d. $p=n$ ), alors $\sigma$ est élémentaire si et seulement si son volume est impair.

Proposition 4.1. (Itenberg [I]) Si $\sigma$ est un p-simplexe élémentaire dans $\left(\mathbb{R}_{+}\right)^{n}$ qui n'appartient pas un hyperplan de coordonnées, alors $\sigma$ a exactement $2^{n}-2^{n-p}$ copies symétriques non vides.

Preuve. Comme $\sigma$ est élémentaire, la matrice $\mathcal{M}_{\sigma}$ est de rang maximal égal à $p$. L'espace des solutions de $(\star)$ est un donc un $\mathbb{Z} / 2$-espace affine de dimension $n-p$ et possède exactement $2^{n-p}$ solutions distinctes.

Les deux lemmes suivant donnent des résultats similaires pour certains simplexes non élémentaires.

Lemme 4.1. Supposons ici $n \leq 3$. Si $\sigma$ est un p-simplexe dans $\left(\mathbb{R}_{+}\right)^{n}$ qui n'appartient pas un hyperplan de coordonnées et tel que $|\sigma|$ soit 
l'union des sommets de $\sigma$ et du milieu $v$ de l'une des ses arêtes $e_{v}$, alors, toutes les copies symétriques de $\sigma$ sont non vides si les sommets de $e_{v}$ ont des signes différents, sinon $\sigma$ a exactement $2^{n}-2^{n-p+1}$ copies symétriques non vides.

Preuve. Supposons que $v_{0}$ et $v_{1}$ soient les sommets de $e_{v}$. Puisque le milieu $v$ de $e_{v}$ est un point entier, on a $\overline{v_{1}}+\overline{v_{0}}=0$ dans $(\mathbb{Z} / 2)^{n}$, et donc la matrice $\mathcal{M}_{\sigma}$ est de rang au plus $p-1$. Il est facile de voir que $|\sigma|=\left\{v_{0}, \cdots, v_{p}\right\} \cup\{v\}$ et $p \leq 3$ impliquent que ce rang est égal à $p-1$. Il reste à remarquer que $(\star)$ a des solutions si et seulement si $\overline{d\left(v_{0}\right)}+\overline{d\left(v_{1}\right)}=0$.

Lemme 4.2. Si $\sigma$ est un tétraèdre maximal de volume pair contenu dans $\left(\mathbb{R}_{+}\right)^{3}$, alors, les huit copies symétriques de $\sigma$ sont non vides si le produit des signes aux sommets de $\sigma$ est négatif, sinon $\sigma$ a exactement six copies symétriques non vides.

Preuve. Le fait que le volume de $\sigma$ soit pair implique que la $(3 \times 3)$ matrice $\mathcal{M}_{\sigma}$ associée est de rang au plus deux. Le fait que $\sigma$ soit maximal (et de dimension trois) implique que la seule relation non triviale entre les vecteurs lignes de $\mathcal{M}_{\sigma}$ est donnée par $\overline{v_{0}}+\overline{v_{1}}+\overline{v_{2}}+\overline{v_{3}}=0 \in(\mathbb{Z} / 2)^{3}$. Par conséquent, le rang de $\mathcal{M}_{\sigma}$ est deux. Il reste à voir que le système $(\star)$ admet des solutions si et seulement si $\overline{d\left(v_{0}\right)}+\overline{d\left(v_{1}\right)}+\overline{d\left(v_{2}\right)}+\overline{d\left(v_{3}\right)}=0$.

On se donne une triangulation $\tau$ de $Q_{k}$ et une distribution de signes $D$ aux sommets de $\tau$. Soient $\tilde{S}_{k}$ la surface linéaire par morceaux dans $\tilde{Q}_{k}$ construite comme dans la section 3 à partir de $\tau$ et $D$ et, si $\tau$ est convexe, $\mathcal{C}_{k}$ une T-surface associée. Le résultat qui suit est formulé en terme de $\tilde{S}_{k}$ puisque sa preuve ne nécéssite pas l'hypothèse de convexité de $\tau$, néanmoins, moyennant cette dernière hypothèse, on pourra y remplacer $\tilde{S}_{k}$ par $\mathbb{R} \mathcal{C}_{k}$. On pose $m=2 k$.

Proposition 4.2. Si la triangulation $\tau$ est maximale, alors la caractéristique d'Euler de $\tilde{S}_{k}$ vérifie

$$
\chi\left(\tilde{S}_{k}\right)=-\frac{m^{3}}{3}+\frac{4 m}{3}+\sum_{\gamma \in \tau}\left(\operatorname{vol}(\gamma)-\varepsilon_{\gamma}\right),
$$


où $\varepsilon_{\gamma}$ vaut, 1 si $\gamma$ est un tétraèdre de volume impair, 2 si $\gamma$ est un tétraèdre de volume pair dont le produit des signes aux sommets est positif, et 0 sinon.

Preuve. La triangulation $\tau$ étant maximale, tous ses simplexes sont élémentaires exceptés les tétraèdres de volume pair. Pour $n \leq 3$ et $p \leq 2$, on note $l_{p}^{n}$ le nombre de $p$-simplexes de $\tau$ qui sont contenus dans une $n$-face de $Q_{k}$ mais non contenus dans une $(n-1)$-face de $Q_{k}$ (la 3face est $Q_{k}$ ). On note $e$ le nombre de tétraèdres de $\tau$ de volume impair et $e^{+}$(resp. $e^{-}$) le nombre de tétraèdres de volume pair de $\tau$ dont le produit des signes aux sommets est positif (resp. négatif). On pose $E=$ $e+e^{+}+e^{-}$. On a facilement $l_{1}^{1}=6 m, l_{1}^{2}=6 m^{2}, l_{1}^{3}=E+\frac{m^{3}}{6}-3 m^{2}-\frac{25 m}{6}$, $l_{2}^{2}=4 m^{2}$ et $l_{2}^{3}=2 E-2 m^{2}$. En utilisant la proposition 4.1 et le lemme 4.2 , on obtient que la surface $\tilde{S}_{k}$ est constituée de $4 E+2 m^{3} / 3+4 m / 3$ sommets, $12 E$ arêtes et $6 e^{+}+7 e+8 e^{-}$cellules de dimension deux. La formule d'Euler implique alors $\chi\left(\tilde{S}_{k}\right)=-\frac{m^{3}}{3}+\frac{4 m}{3}+m^{3}-\left(e+2 e^{+}\right)$. Il reste à utiliser l'égalité $\operatorname{vol}\left(Q_{k}\right)=m^{3}$.

Remarque 4.1. Toute surface $\mathcal{C}_{k}$ pouvant se déformer sur une surface $X_{m}$ dans $\mathbb{C} P^{3}$, il s'ensuit que de telles surfaces ont les mêmes nombres de Hodge. En particulier, on a $\sigma\left(\mathcal{C}_{k}\right)=-\frac{m^{3}}{3}+\frac{4 m}{3}$ (voir section 1). Comme corollaire de la proposition 4.2 on obtient donc l'inégalité $\chi\left(\mathbb{R} \mathcal{C}_{k}\right) \geq \sigma\left(\mathcal{C}_{k}\right)$ pour une T-surface $\mathcal{C}_{k}$ issue d'une triangulation maximale. En utilisant l'inégalité de Smith-Thom, on voit que l'inégalité $\chi\left(\mathbb{R C}_{k}\right) \geq \sigma\left(\mathcal{C}_{k}\right)$ implique $b_{1}\left(\mathbb{R C}_{k}\right) \leq h^{1,1}\left(\mathcal{C}_{k}\right)$. Autrement dit la proposition 4.2 implique que l'inégalité de Viro est vraie pour une T-surface $\mathcal{C}_{k}$ issue d'une triangulation maximale (voir $[\mathrm{I}]$ ). On doit donc utiliser des triangulations non maximales si l'on veut espérer obtenir des contreexemples à l'inégalité de Viro.

Un segment $I$ dans $\mathbb{R}^{n}$ est de longueur 2 si $|I|$ est constitué des deux sommets et du milieu de $I$. Supposons maintenant que $\tau$ contienne une arête $\gamma$ de longueur 2 et de milieu $v$. On peut raffiner $\tau$ suivant $v$ en subdivisant chaque simplexe de $\tau$ ayant $\gamma$ comme arête en deux simplexes ayant $v$ comme sommet. Appelons flip cette modification de triangulation (c'est un exemple d'une modification de triangulation le long d'un circuit comme défini dans [G-K-Z]). Soient $\tau_{v}$ la triangulation 
résultant de ce flip et $D_{v}$ une distribution de signes aux sommets de $\tau_{v}$ qui coincide avec $D$ sur les sommets de $\tau$ (on choisit un signe pour $v$ ). On note $\tilde{S}_{k}^{v}$ la surface linéaire par morceaux dans $\tilde{Q}_{k}$ construite à partir de $\tau_{v}$ et $D_{v}$.

Proposition 4.3. Si les sommets de $\gamma$ ont des signes différents, alors les surfaces $\tilde{S}_{k}$ et $\tilde{S}_{k}^{v}$ sont homéomorphes. Supposons que les sommets de $\gamma$ aient le même signe et que tous les simplexes de $\tau_{v}$ ayant $v$ comme sommet soient maximaux. Si $\gamma$ est contenue dans une $n$-face de $Q_{k}$ mais non contenue dans une $(n-1)$-face de $Q_{k}$, alors

$$
\chi\left(\tilde{S}_{k}\right)-\chi\left(\tilde{S}_{k}^{v}\right)=e+2 e^{+}-2^{n},
$$

où e est le nombre de tétraèdres élémentaires de $\tau_{v}$ ayant $v$ comme sommet et $e^{+}$est le nombre de tétraèdres de volume pair de $\tau_{v}$ ayant $v$ comme sommet et dont le produit des signes aux sommets est positif.

Preuve. Soient $v_{0}$ et $v_{1}$ les sommets de $\gamma$. Supposons que $v_{0}$ et $v_{1}$ aient des signes différents. Alors, puisque $\overline{v_{0}}=\overline{v_{1}}$ dans $(\mathbb{Z} / 2)^{3}$, les copies symétriques $r\left(v_{0}\right)$ et $r\left(v_{1}\right)$ ont des signes différents pour tout $r \in \mathcal{R}^{3}$. Il est alors facile de voir que $\tilde{S}_{k}^{v}$ est homéomorphe à $\tilde{S}_{k}$.

Supposons maintenant que $v_{0}$ et $v_{1}$ aient le même signe et montrons la proposition dans le cas $n=3$ (les autres cas sont similaires) c.a.d. $\gamma$ est contenu à l'intérieur de $Q_{k}$. Pour calculer la différence $\chi\left(\tilde{S}_{k}\right)-$ $\chi\left(\tilde{S}_{k}^{v}\right)$ on applique comme dans la preuve de la proposition 4.2 la formule d'Euler à chacune des surfaces $\tilde{S}_{k}$ et $\tilde{S}_{k}^{v}$. Bien sur il suffit ici de considérer les cellules contenues dans les copies symétriques, des simplexes de $\tau$ ayant $\gamma$ comme arête d'une part, et des simplexes de $\tau_{v}$ ayant $v$ comme sommet d'autre part. Soit $e^{-}$le nombre de tétraèdres de volume pair de $\tau_{v}$ ayant $v$ comme sommet et dont le produit des signes aux sommets est négatif. Posons $E=e+e^{+}+e^{-}$. Les simplexes de $\tau$ ayant $\gamma$ comme arête sont $\frac{E}{2}$ tétraèdres, $\frac{E}{2}$ triangles et $\gamma$. En utilisant la proposition 4.3 , on obtient que les copies symétriques de ces simplexes contiennent exactement $3 E$ cellules de dimension deux et $2 E$ arêtes de $\tilde{S}_{k}$. Les simplexes de $\tau_{v}$ ayant $v$ comme sommets sont $E$ tétraèdres, $\frac{3 E}{2}$ cellules de dimension deux, $\frac{E}{2}+2$ arêtes et $v$. En utilisant la proposition 4.1 et le lemme 4.2, on obtient que les copies symétriques de ces simplexes contiennent $6 e^{+}+7 e+8 e^{-}$cellules de dimension deux, $9 E$ arêtes et $2 E+8$ sommets de $\tilde{S}_{k}^{v}$. Finalement, $\chi\left(\tilde{S}_{k}\right)-\chi\left(\tilde{S}_{k}^{v}\right)=3 E-2 E-\left(6 e^{+}+\right.$ $\left.7 e+8 e^{-}-9 E+2 E+8\right)=e+2 e^{+}-8$. 
Remarque 4.2. Avec les mêmes hypothèses que dans la proposition précédente, on voit facilement que l'on a $\chi\left(\tilde{S}_{k}\right)<\chi\left(\tilde{S}_{k}^{v}\right)$ si et seulement si

- les sommets de $\gamma$ ont le même signe,

- $\gamma$ est contenue à l'intérieur de $Q_{k}$,

- les tétraèdres de $\tau$ ayant $\gamma$ comme arête sont trois tétraèdres de volume deux.

En outre si ces trois conditions sont remplies, on a $\chi\left(\tilde{S}_{k}\right)=\chi\left(\tilde{S}_{k}^{v}\right)-2$. En particulier, si $\chi\left(\tilde{S}_{k}^{v}\right) \leq-\frac{m^{3}}{3}+\frac{4 m}{3}$ (ce qui est vérifié si $\tau_{v}$ est par exemple une triangulation primitive, d'après la proposition 4.2), alors $\chi\left(\tilde{S}_{k}^{v}\right)<-\frac{m^{3}}{3}+\frac{4 m}{3}$ et l'on peut espérer, moyennant l'hypothèse de convexité de $\tau_{v}$, qu'une T-surface $\mathcal{C}_{k}^{v}$ associée à $\tau_{v}$ et $D_{v}$ soit un contreexemple à l'inégalité de Viro (voir la remarque 4.1).

\section{Constructions}

Soient $\left(y_{1}, y_{2}, y_{3}\right)$ des coordonnées de $\mathbb{R}^{3}$. Notons $s$ la réflection de $\mathbb{R}^{3}$ par rapport au plan passant par les points $(0,0,0),(1,1,0)$ et $(0,0,1)$. Notons $Q^{l}$ le carré obtenu comme intersection du polytope $Q_{k}$ avec le $\operatorname{plan}\left\{y_{3}=l\right\}$ pour $l=0, \cdots, k-1$.

Nous fixons ici $k=3$ et construisons une T-surface $\mathcal{C}_{3}$ dans $\Theta$. Soit $\tau_{0}$ la subdivision polyédrale de $Q_{3}$ formée du cone $C$ de sommet $(0,0,2)$ sur $Q^{0} ;$ du tétraèdre $T_{1}$ de sommets $(0,0,2),(6,6,0),(4,0,1)$, $(6,0,0) ;$ du tétraèdre $T_{2}$ de sommets $(0,0,2),(6,6,0),(4,4,1),(4,0,1)$; du tétraèdre $T_{3}$ de sommets $(0,0,2),(2,0,2),(4,4,1),(4,0,1)$; des tétraèdres $s\left(T_{i}\right), i=1,2,3$ et des deux cones de sommets $(0,0,3)$ et $(4,4,1)$ sur $Q^{2}$ (figure 2). La triangulation $\tau$ de $Q_{3}$ que nous allons utiliser est obtenue en raffinant $\tau_{0}$ de la manière suivante.

Considérons la triangulation du quadrangle $q$ de sommets $(3,0,0)$, $(1,3,0),(3,6,0),(5,3,0)$ montrée sur la figure 2 . Il est possible d'étendre cette triangulation en une triangulation convexe maximale de $Q^{0}$. Fixons une telle triangulation de $Q^{0}$ et triangulons le cone $C$ en prenant les cones 
de sommet $(0,0,2)$ sur les triangles de la triangulation de $Q^{0}$. La triangulation de $C$ ainsi obtenue n'est pas maximale: chacun des points de $\mid Q^{1} \cap$ $C \mid$ est le milieu d'une arête de longueur 2. Raffinons la triangulation de $C$ suivant chacun des points de $\left|Q^{1} \cap C\right| \backslash\{(1,1,1),(2,1,1),(2,2,1),(1,2,1)\}$ en utilisant le flip décrit dans la section 4 (Proposition 4.3). Finalement triangulons de l'unique manière possible chacun des autres polytopes de $\tau_{0}$ de telle sorte que chacun des tétraèdres de $\tau$ non contenu dans le cone $C$ soit maximal.

Pour voir que la triangulation $\tau$ est convexe, on peut noter que la subdivision $\tau_{0}$ et les triangulations induites par $\tau$ de chacun des 3-polytopes de $\tau_{0}$ sont convexes. Soient $\nu_{0}: Q_{3} \rightarrow \mathbb{R}$ une fonction certifiant la convexité de $\tau_{0}$ et $\mu: Q_{3} \rightarrow \mathbb{R}$ une fonction affine par morceaux telle que la restriction de $\mu$ sur chacun des 3-polytopes de $\tau_{0}$ est une fonction certifiant la convexité de la triangulation induite par $\tau$. Alors il n'est pas difficile de voir que pour $\epsilon \in \mathbb{R}$ suffisamment proche de zéro la fonction $\nu_{0}+\epsilon \cdot \mu$ certifie la convexité de $\tau$.

On définit maintenant une distribution de signes $D: V(\tau) \rightarrow \pm 1$. Pour les sommets appartenant à $|q|$, on utilise la distribution de signes montrée sur la figure 2, puis on attribue le signe -1 à chacun des six sommets contenus à l'interieur de $Q_{3}$, et +1 à chacun des sommets restants.

Théorème 5.1. Il existe une sextique réelle $X_{6}$ dans $\mathbb{C} P^{3}$ dont l'ensemble des points réels $\mathbb{R} X_{6}$ est homéomorphe à l'union disjointe

$$
6 S \coprod S_{2} \coprod S_{42}
$$

de six sphères, une sphère avec deux anses et une sphère avec 42 anses. Une telle surface est une $(M-2)$-surface vérifiant

$$
b_{1}\left(\mathbb{R} X_{6}\right)=h^{1,1}\left(X_{6}\right)+2=88 .
$$

Preuve. Soient $\tilde{S}_{3}$ la surface linéaire par morceaux dans $\tilde{Q}_{3}$ construite comme dans la section 3 à partir de la paire $(\tau, D)$ décrite plus haut et $\mathcal{C}_{3}$ une T-surface associée. On calcule dans un premier temps la caractéristique d'Euler de $\mathbb{R} C_{3}$.

Raffinons $\tau$ suivant les points $(1,1,1),(2,1,1),(2,2,1),(1,2,1)$ de manière à obtenir une triangulation maximale $\tau^{\prime}$ de $Q_{3}$. Étendons arbitrairement la distribution de signes $D$ en une distribution de signes 

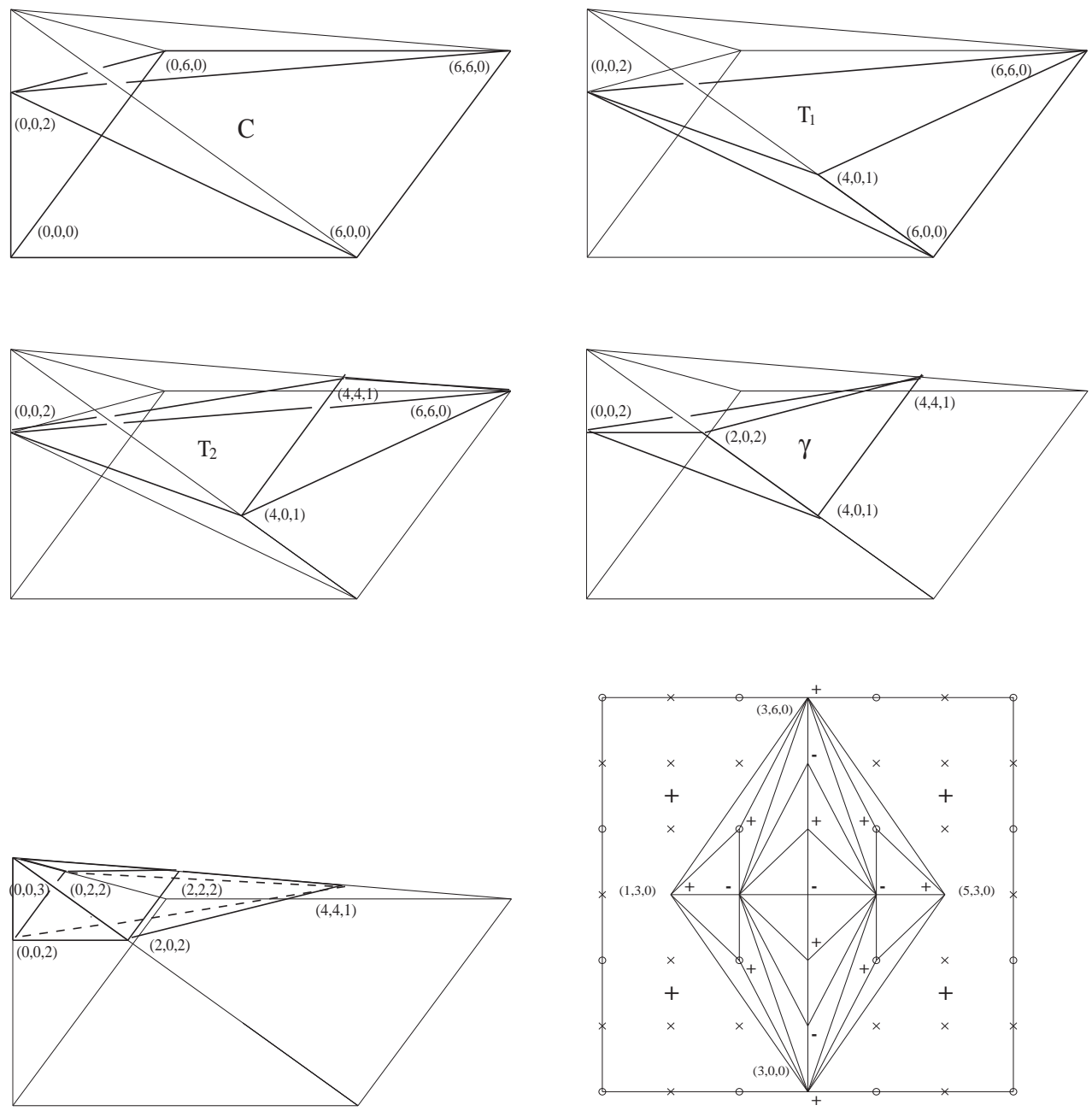

Figure 2: Triangulation de $Q_{3}$. 
$D^{\prime}$ sur les sommets de $\tau^{\prime}$. Soit $\tilde{S}_{3}^{\prime}$ la surface linéaire par morceaux dans $\tilde{Q}_{3}$ construite à partir de la paire $\left(\tau^{\prime}, D^{\prime}\right)$. La triangulation $\tau^{\prime}$ contient deux types de tétraèdres: des tétraèdres primitifs et des tétraèdres maximaux de volume deux contenus dans le cone $C$ et dont le produit des signes aux sommets est positif. La proposition 4.2 implique alors $\chi\left(\tilde{S}_{3}^{\prime}\right)=\sigma\left(\mathcal{C}_{3}\right)=-64$. Revenons maintenant à la T-surface $\mathcal{C}_{3}$. En utilisant successivement quatre fois la proposition 4.3, on obtient $\chi\left(\tilde{S}_{3}\right)=\chi\left(\tilde{S}_{3}^{\prime}\right)-8$ (on se trouve à chaque fois dans la situation décrite dans la remarque 4.2$)$ et finalement

$$
\chi\left(\mathbb{R C}_{3}\right)=-72 .
$$

Chacun des six sommets de $\tau$ contenu à l'intérieur de $Q_{3}$ a le signe -1 alors que tous ses sommets voisins ont le signe +1 (un point $b$ est dit voisin du point $a$ si le segment $[a, b]$ est une arête de la triangulation). On obtient donc six composantes connexes de $\tilde{S}_{3}$ contenues à l'intérieur de $Q_{3}$ qui sont homéomorphes à une sphère. Une autre composante connexe de $\tilde{S}_{3}$ est contenue dans l'union du cone $C$ et de sa copie symétrique par rapport au plan $\left\{y_{3}=0\right\}$, cette composante est homéomorphe à une sphère avec deux anses. La surface $\tilde{S}_{3}$ a au moins une composante connexe supplémentaire puisqu'elle intersecte le plan d'axe $\left\{y_{2}=0\right\}$. En résumé, par la Proposition 3.1, la surface $\mathbb{R C}_{3}$ a au moins huit composantes connexes, six d'entre elles sont homéomorphes à une sphère, une autre est homéomorphe à une sphère avec deux anses. En utilisant $\chi\left(\mathbb{R C}_{3}\right)=-72$ et l'inégalité de smith-Thom $b_{*}\left(\mathbb{R} \mathcal{C}_{3}\right) \leq 108$, on obtient $b_{0}\left(\mathbb{R C}_{3}\right) \leq 9$ et $b_{0}\left(\mathbb{R C}_{3}\right)=9$ si et seulement si $\mathcal{C}_{3}$ est une M-surface. La congruence de Rokhlin permet alors d'interdire le cas $b_{0}\left(\mathbb{R C}_{3}\right)=9$. Par conséquent on a $b_{0}\left(\mathbb{R C}_{3}\right)=8$. Sachant $\chi\left(\mathbb{R C}_{3}\right)=-72$, on détermine la topologie de la dernière composante connexe de $\mathbb{R} C_{3}$ pour finalement obtenir

$$
\mathbb{R C}_{3} \simeq 6 S \coprod S_{2} \coprod S_{42}
$$

Il reste à utiliser la proposition 2.1 pour montrer l'existence d'une sextique $X_{6}$ dans $\mathbb{C} P^{3}$ telle que $\mathbb{R} X_{6} \simeq \mathbb{R} C_{3}$.

Soit $k$ un entier tel que $k \geq 4$. Nous construisons ici une T-surface $\mathcal{C}_{k}$ dans $\Theta$. Soient $\wedge$ l'union dans $Q_{k}$ du triangle de sommets $(0,0,2)$, 


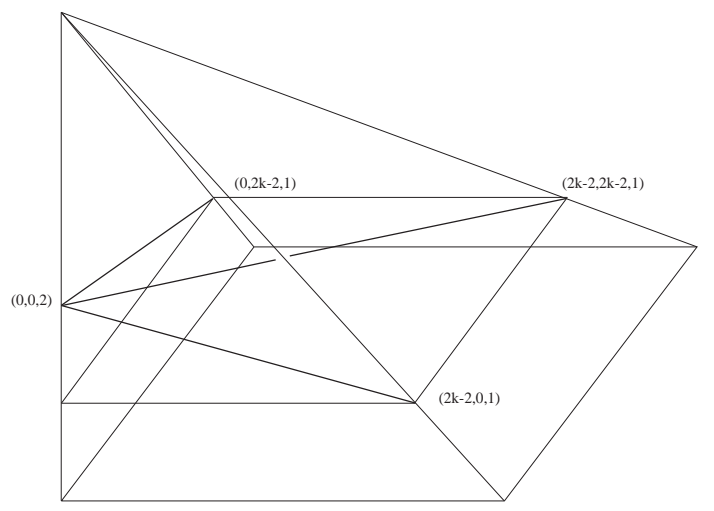

Figure 3: Séparation par $\wedge$.

$(2 k-2,0,1),(2 k-2,2 k-2,1)$ et de son image symétrique par $s$. Notons $\Lambda$ (resp. $\pi$ ) la partie de $Q_{k}$ située en dessous (resp. au dessus) de $\wedge$ (voir figure 3 ).

On subdivise dans un premier temps (voir figure 4$) \wedge$ par le cone $C$ de sommets $(0,0,2)$ sur $Q^{0} ;$ le tétraèdre $T_{1}$ de sommets $(k+1,0,1)$, $(0,0,2),(2 k, 2 k, 0),(2 k, 0,0)$; le tétraèdre $T_{2}$ de sommets $(k+1,0,1)$, $(2 k-2,0,1),(2 k, 2 k, 0),(2 k, 0,0)$, les tétraèdres $s\left(T_{1}\right), s\left(T_{2}\right)$ et les deux cones de sommets $(0,0,2)$ et $(2 k, 2 k, 0)$, respectivement, sur la partie $H$ de $Q^{1}$ montrée sur la figure 4. Puis on raffine cette subdivision comme suit. On triangule le cone $C$ comme dans le cas $k=3$ c.a.d. on étend la triangulation, montrée dans la figure 2 , du quadrangle $q$ de sommets $(3,0,0),(1,3,0),(3,6,0),(5,3,0)$ en une triangulation convexe maximale de $Q^{0}$, on prend tous les cones de sommet $(0,0,2)$ sur les triangles de la triangulation ainsi obtenue puis on raffine suivant tous les points de $\left|Q^{1} \cap C\right| \backslash\{(1,1,1),(2,1,1),(2,2,1),(1,2,1)\}$. Actuellement le triangle de sommets $(0,0,2),(2 k, 2 k, 0),(2 k, 0,0)$ et son image symétrique par $s$ sont triangulés. On étend ces triangulations de la seule manière possible en des triangulations maximales de $T_{1}$ et de $s\left(T_{1}\right)$. On triangule de la seule manière possible $T_{2}$ et $s\left(T_{2}\right)$ par des tétraèdres maximaux. Finalement, on choisit une triangulation convexe maximale de $H$ et on étend cette triangulation en une triangulation des deux cones sur $H$.

Nous triangulons maintenant $\pi$. On triangule la partie située entre $\wedge$ et $Q^{2}$ par le cone de sommet $(2 k-2,2 k-2,1)$ sur $Q^{2} ;$ le tétraèdre de 

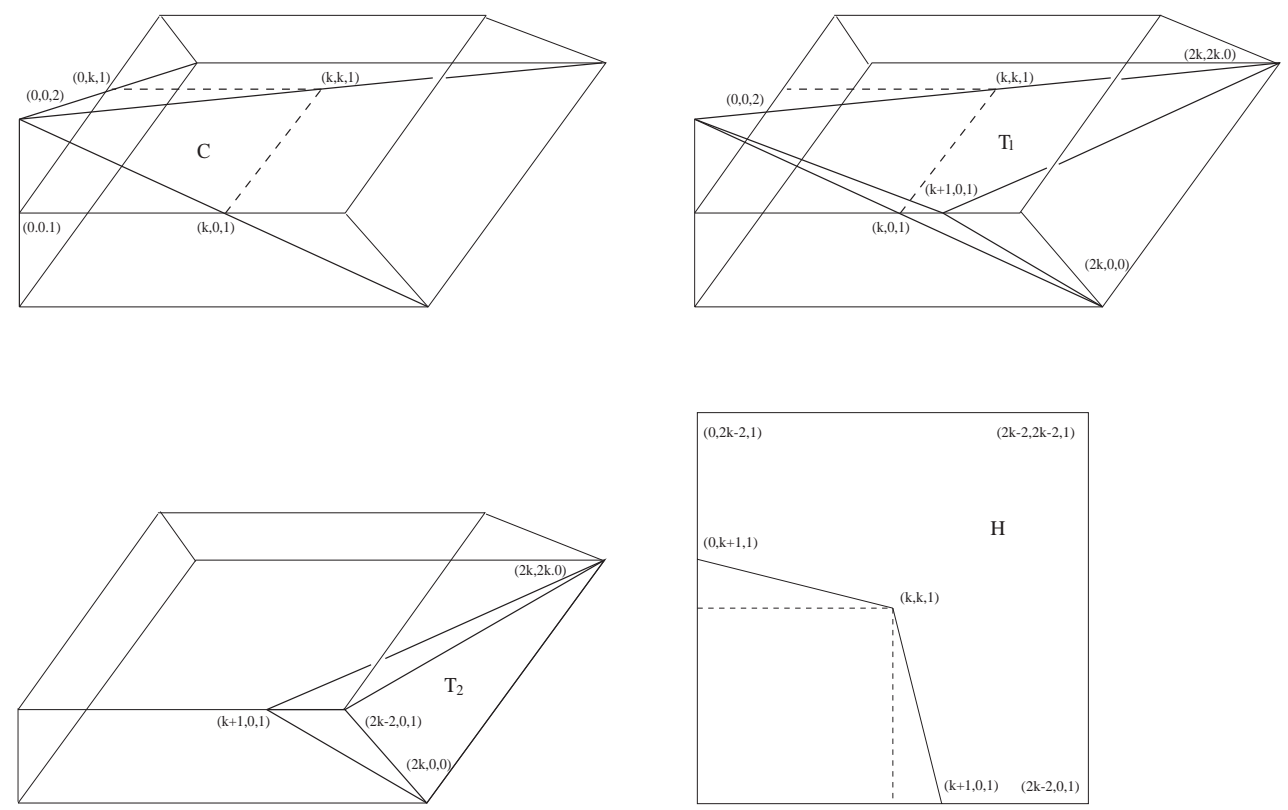

Figure 4: Subdivision de $\underline{\wedge}$.

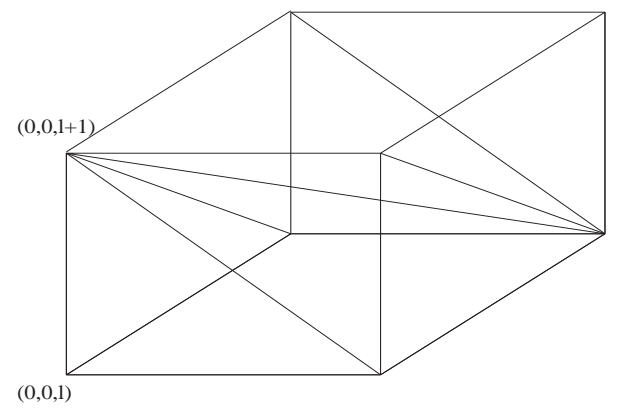

Figure 5: Triangulation entre $Q^{l}$ et $Q^{l+1}$.

(5) 
sommets $(2 k-2,0,1),(2 k-2,2 k-2,1),(0,0,2),(2 k-4,0,2)$ et son image symétrique par $s$. Puis, pour tout entier $l$ tel que $2 \leq l \leq k-2$, on triangule la partie située entre $Q^{l}$ et $Q^{l+1}$ (figure 5) par le cone de sommet $(0,0, l+1) \operatorname{sur} Q^{l}$; le cone de sommet $(2 k-2 l, 2 k-2 l, l) \operatorname{sur} Q^{l+1}$; le tétraèdre de sommets $(2 k-2 l, 0, l),(2 k-2 l, 2 k-2 l, l),(0,0, l+1)$, $(2 k-2 l-2,0, l+1)$ et son image symétrique par $s$. Il reste à prendre le cone de sommets $(0,0, k)$ sur $Q^{k-1}$. Finalement, nous raffinons la triangulation de $\pi$ obtenue en une triangulation maximale en utilisant une triangulation convexe maximale arbitraire de chacun des carrés $Q^{l}$ avec $2 \leq l \leq k-1$, on suppose seulement que la triangulation de $Q^{2}$ choisie contient le triangle de sommets $(0,0,2),(1,0,2)$ et $(0,1,2)$ (nous utiliserons le fait que $(0,0,2)$ n'est pas voisin d'un point de $\left|Q_{k} \cap Q^{2}\right|$ contenu à l'intérieur de $Q_{k}$ ).

Par des arguments similaires à ceux employés dans la cas $k=3$ précédent, on peut voir que la triangulation $\tau$ de $Q_{k}$ que l'on vient de décrire est convexe. On définit maintenant une distribution de signes $D: V(\tau) \rightarrow \pm 1$.

Pour les sommets appartenant à $|q|$, on utilise la distribution de signes montrée sur la figure 2 , on distribue le signe +1 aux autres sommets appartenant à $Q^{0}$.

On distribue le signe -1 à chacun des sommets de $\tau$ appartenant à $\left|C \cap Q^{1}\right|$ et le signe +1 au point $(0,0,2)$.

Pour les autres sommets de $\tau$, on distribue le signe -1 au point $\left(y_{1}, y_{2}, y_{3}\right)$ si $y_{1} \equiv y_{2} \equiv k \bmod 2$, et le signe +1 sinon.

Théorème 5.2. Pour tout entier pair $m \geq 6$, il existe une surface réelle $X_{m}$ dans $\mathbb{C} P^{3}$ telle que $b_{1}\left(\mathbb{R} X_{m}\right)>h^{1,1}\left(X_{m}\right)$.

Preuve. Le cas $m=6$ est donné par le théorème 5.1. Supposons maintenant $m=2 k \geq 8$. La preuve est similaire à celle du théorème 5.1. Soient $\tilde{S}_{k}$ la surface linéaire par morceaux dans $\tilde{Q}_{k}$ construite comme dans la section 3 à partir de la paire $(\tau, D)$ décrite plus haut et $\mathcal{C}_{k}$ une T-surface associée.

On calcule tout d'abord la caractéristique d'Euler de $\mathbb{R} \mathcal{C}_{k}$. L'ensemble des sommets de $\tau$ est $\left|Q_{k}\right|$ moins les points $(1,1,1),(2,1,1),(2,2,1)$ et $(1,2,1)$, ces points étant les milieux d'arêtes de longueur 2 de $\tau$. Un tétraèdre de $\tau$ qui n'a pas l'une de ces arêtes de longueur 2 comme arête est soit primitif soit maximal de volume deux et de produit des signes positif aux sommets. En utilisant les propositions 4.2 et 4.3 , on obtient 
$\chi\left(\mathbb{R} \mathcal{C}_{k}\right)=-\frac{m^{3}}{3}+\frac{4 m}{3}-8$.

La triangulation $\tau$ possède $h^{2,0}\left(\mathcal{C}_{k}\right)-4=\frac{m^{3}}{6}-m^{2}+\frac{11 m}{6}-5$ sommets contenus à l'intérieur de $Q_{k}$. Soit $v$ l'un de ces sommets, nous montrons qu'il existe une et une seule copie symétrique $r(v)$ dont tous les voisins dans $\tau^{*}$ ont le signe opposé à celui de $r(v)$. On obtient alors $\frac{m^{3}}{6}-$ $m^{2}+\frac{11 m}{6}-5$ composantes connexes de $\tilde{S}_{k}$ homéomorphes à une sphère. Si $v$ appartient à $\left|C \cap Q^{1}\right|$ alors $v$ a le signe -1 tandis que chacun de ses voisins a le signe +1 , la copie symétrique à considérer est $r(v)=$ $v$. Soit maintenant $v$ un sommet de $\tau$ contenu à l'intérieur de $Q_{k}$ et n'appartenant pas à $\left|C \cap Q^{1}\right|$. Le point $v$ appartient à un certain $\left|Q^{l}\right|$, ses voisins sont des points de $\left|Q^{l}\right|,(0,0, l+1)$ et $(2 k-2 l-2,2 k-2 l-2, l-1)$. Puisque deux voisins de $v$ ayant même réduction dans $(\mathbb{Z} / 2)^{3}$ ont le même signe (rappelons que le point $(0,0,2)$ n'est pas voisin d'un point de $\left|Q_{k} \cap Q^{2}\right|$ contenu à l'intérieur de $\left.Q_{k}\right)$, il suffit de considérer $(0,0, l+1)$ et trois voisins $v_{1}, v_{2}, v_{3}$ de $v$ appartenant à $\left|Q^{l}\right|$ dont les réductions dans $(\mathbb{Z} / 2)^{3}$ sont deux à deux distinctes. On peut de plus choisir ces points de telle sorte que, par exemple, $v_{1}$ et $v_{2}$ soient voisins, auquel cas, le tétraèdre de sommets $v, v_{1}, v_{2}$ et $(0,0, l+1)$ est un tétraèdre primitif de $\tau$ (le triangle de sommets $v, v_{1}$ et $v_{2}$ est maximal). Exactement de la même manière que dans la preuve de la proposition 4.1, on montre qu'il existe une unique symétrie $r_{v} \in \mathcal{R}^{3}$ pour laquelle $r_{v}\left(v_{1}\right), r_{v}\left(v_{2}\right)$ et $r_{v}((0,0, l+1))$ ont tous le signe opposé à celui de $r_{v}(v)$. Il reste à voir que $r_{v}\left(v_{3}\right)$ a également le signe opposé à celui de $r_{v}(v)$. Pour cela on remarque que $\bar{v}+\overline{v_{1}}+\overline{v_{2}}+\overline{v_{3}}=0$ dans $(\mathbb{Z} / 2)^{3}$, ce qui implique que le produit des signes des points $r(v), r\left(v_{1}\right), r\left(v_{2}\right)$ et $r\left(v_{3}\right)$ est le même pour toute symétrie $r \in \mathcal{R}^{3}$. En particulier puisque le produit des signes des points $v, v_{1}, v_{2}$ et $v_{3}$ est négatif, il en va de même pour leurs copies symétriques par $r_{v}$.

La surface $\tilde{S}_{k}$ possède donc $\frac{m^{3}}{6}-m^{2}+\frac{11 m}{6}-5$ composantes connexes homéomorphes à une sphère. On obtient une autre composante homéomorphe à une sphère avec deux anses qui est contenue dans l'union du cone $C$ et de sa copie symétrique par rapport au plan $\left\{y_{3}=0\right\}$. Il existe une composante connexe supplémentaire de $\tilde{S}_{k}$ intersectant le plan d'axe $\left\{y_{2}=0\right\}$. En résumé, par la Proposition 3.1, la surface $\mathbb{R C}_{k}$ au moins $\frac{m^{3}}{6}-m^{2}+\frac{11 m}{6}-3$ composantes connexes, $\frac{m^{3}}{6}-m^{2}+\frac{11 m}{6}-5$ d'entre elles sont homéomorphes à une sphère, une autre est homéomorphe à une sphère avec deux anses. Comme dans la preuve du théorème 5.1 , on 
prouve, en utilisant l'inégalité de Smith-Thom, la congruence de Rokhlin et l'égalité $\chi\left(\mathbb{R C}_{k}\right)=-\frac{m^{3}}{3}+\frac{4 m}{3}-8$, que la surface $\mathbb{R} \mathcal{C}_{k}$ ne possède pas d'autres composantes connexes. Puis via la caractéristique d'Euler, on obtient que la dernière composante connexe de $\mathbb{R C}_{k}$ est homéomorphe à une sphère avec $\frac{m^{3}}{3}-m^{2}+\frac{7 m}{6}-1$ anses. Finalement la surface $\mathbb{R} \mathcal{C}_{k}$ est homéomorphe à

$$
\left(\frac{m^{3}}{6}-m^{2}+\frac{11 m}{6}-5\right) S \coprod S_{2} \coprod S_{\frac{m^{3}}{3}-m^{2}+\frac{7 m}{6}-1} .
$$

En particulier, on a $b_{1}\left(\mathbb{R C}_{k}\right)=h^{1,1}\left(\mathcal{C}_{k}\right)+2$. Il reste à utiliser la proposition 2.1 pour obtenir l'existence d'une surface $X_{m}$ dans $\mathbb{C} P^{3}$ avec la même propriété.

Remarque 5.1. On peut modifier comme dans [I] les triangulations de $Q_{k}$ utilisées ici afin d'obtenir, après déformation, des surfaces $X_{m}$ vérifiant $b_{1}\left(\mathbb{R} X_{m}\right)=h^{1,1}\left(X_{m}\right)+a \cdot m^{3}+O\left(m^{2}\right)$ où $a$ est une constante strictement positive. Mais il parait clair que l'on ne pourra pas de cette manière améliorer un résultat de [B2] où une famille de surfaces $X_{m}$ vérifiant $b_{1}\left(\mathbb{R} X_{m}\right)>\left(\frac{13}{18}-\epsilon\right) \cdot m^{3}$ pour $m$ suffisamment grand est construite pout tout $\epsilon>0$ donné.

\section{References}

[B1] F. Bihan, Betti numbers of real numerical quintic surfaces, à paraitre dans Amer. Math. Soc. Transl. (2), Rokhlin's memorial volume, (2000).

[B2] F. Bihan, Asymptotics of Betti numbers of real algebraic surfaces, preprint, université de Genève (Suisse), (2000).

[D-K] A. Degtyarev, V. M. Kharlamov, Topological properties of real algebraic varieties: Rokhlin's way, à paraitre, (2000).

[G-K-Z] I. Gelfand, M. Kapranov and A. Zelevinsky, Discriminants, resultants and multidimensional determinants, Birkhauser, Boston, (1994).

[H] E. Horikawa, Deformations of Sextic Surfaces, Topology 32, ${ }^{0} .4$, (1993), 757-772.

[I] I. Itenberg, Topology of real algebraic T-surfaces, Revista Matematica, $\mathrm{N}^{0}$. supp, 10, (1997). 
F. Bihan UNE SEXTique DE L'ESPACE PRoJeCtif RÉEl AVEC UN GRAND ...

[I-K] I. Itenberg, V. M. Kharlamov, Towards the maximal number of components of a non-singular surface of degree 5 in $\mathbb{R} P^{3}$, Amer. Math. Soc. Transl. (2) 173 (1996), 111-118.

[K1] V. M. Kharlamov, Topological types of nonsingular surfaces in $\mathbb{R} P^{3}$ of degree 4, Funct. Anal. Appl. 10 (1976), 55-68.

[K2] V. M. Kharlamov, Real algebraic surfaces, Proc. Internat. Congress Math., Helsinky, (1978), 1, 421-428 (in Russian).

[R] J.-J. Risler, Construction d'hypersurfaces réelles [d'apres Viro], Séminaire N. Bourbaki, N . 763, Vol. 1992-93, Novembre 1992.

[V1] O. Viro, Construction of M-surfaces, Funct. Anal. Appl. 13 (1979), $\mathrm{N}^{0} .3$.

[V2] O. Viro, Construction of multicomponent real algebraic surfaces, Sov. Math. Doklady 20 (1979), 991-995.

[V3] O. Viro, Gluing of algebraic hypersurfaces, smoothing of singularities and contruction of curves, Proc. Leningrad Int. Topological Conf. (Leningrad, Aug. 1983), Nauka, Leningrad, 1983, pp. 149-197 (in Russian).

[V4] O. Viro, Real algebraic plane curves: constructions with controlled topology, Leningrad Math. J. 1 (1990), 1059-1134.

[W] G. Wilson, Hilbert's sixteen problem, Topology 17 (1978), Nº . 1, 5374 .

Section de Mathématiques

Université de Genève

2-4 rue du Lièvre

CH-1211 Genève 24

Switzlerland

E-mail: Frederic.Bihan@math.unige.ch

Recibido: 7 de Noviembre de 2000

Revisado: 28 de Noviembre de 2000 